\title{
MEMÓRIA ORGANIZACIONAL: UMA REVISÃO BIBLIOMÉTRICA EM BASES NACIONAIS
}

\author{
Álvaro Guillermo Rojas Lezana ${ }^{1}$ \\ Francisco Antonio Pereira Fialho² \\ Juliana Leonardi ${ }^{3}$ \\ Luciane Camilotti ${ }^{4}$ \\ Rogério Cid Bastos ${ }^{5}$ \\ Telmo José Souto-Maior ${ }^{6}$
}

\begin{abstract}
RESUMO
Tendo em vista a importância que o tema Memória Organizacional (MO) tem assumido dentro das organizações e nas práticas de Gestão do Conhecimento, esta pesquisa tem como objetivo mapear o termo $\mathrm{MO}$, nas bases de publicações nacionais, das áreas de Administração, Ciências Contábeis e Turismo; Psicologia; e Interdisciplinar e também da Base de Dados do Evento EnANPAD. Foi utilizada a técnica bibliométrica na busca sistemática pelo termo $\mathrm{MO}$ nas bases de dados nacionais nos periódicos com Qualis A1, A2, B1, B2 e B3, considerados mais relevantes e na base de dados do EnANPAD, evento mais relevante da área da Administração, para investigar o conceito e aplicação deste termo nas pesquisas nacionais. O processo de desenvolvimento deste estudo constituiu-se em duas etapas: busca sistemática e análise descritiva. Como resultados, a pesquisa mostrou que existe limitações quanto a definição e abordagem do tema MO nas bases nacionais, tanto como construto como aplicação. Estudos sobre o tema são relativamente recentes no Brasil, o que possibilita oportunidades de pesquisas empíricas ou não-empíricas e que possam contribuir para elaboração de construtos e aplicações em âmbito nacional.
\end{abstract}

Palavras-chave: Memória organizacional. Técnica bibliométrica. Gestão do conhecimento.

\footnotetext{
${ }^{1}$ Doutor, e-mail: lezana@deps.ufsc.br

2Doutor, e-mail: fapfialho@gmail.com

${ }^{3}$ Mestre, e-mail: leonardiadm@gmail.com

${ }^{4}$ Doutora, e-mail:Icamilotti@gmail.com

${ }^{5}$ Doutor, e-mail: rogerio@reitoria.ufsc.br

${ }^{6}$ Graduado, e-mail:i telmojsm@yahoo.com.br
} 


\section{INTRODUÇÃO}

O tema memória organizacional se estabelece como um diferencial nas práticas organizacionais e sustenta a execução dos processos gerenciais que priorizam a aplicação do conhecimento para execução de rotinas e procedimentos. Pesquisadores apontam que estudos relativos ao mapeamento do conhecimento, no que tange à criação, armazenamento, compartilhamento e utilização deste nas organizações são relevantes para garantir a fonte de sustentabilidade e vantagem competitiva, por meio dos ativos intelectuais das organizações (DRUCKER, 1993; GRANT, 1996; KOGUT e ZANDER, 1992; NONAKA e TOYAMA, 2002, tradução nossa). Bornemann, et al. (2003, tradução nossa) salientam que maximizar a eficiência e o retorno sobre os ativos de conhecimento da organização é o papel da Gestão do Conhecimento (GC), que apoia e coordena a criação, transferência e aplicação do conhecimento individual, "dando suporte aos processos envolvidos em torno da memória organizacional" ( SASIETA; BEPLLER e PACHECO 2011). Isto é possível, por meio de um processo sistemático, articulado e institucional, apoiado na identificação, geração, compartilhamento e aplicação do conhecimento organizacional (SUTTON e PATKAR, 2009; WU e ONG 2008, tradução nossa).

As organizações são grupos sociais que absorvem e desenvolvem sistemas de conhecimentos para servir a seus propósitos. Ackerman e Halverson (2004, tradução nossa) afirmam que organizações podem ser vistas como sistemas de processamento de informação, no qual interpretações coletivas do conhecimento existem e do qual emergem. Assim, organizações têm uma memória particular: o conhecimento de como fazer as coisas, a forma de abordar problemas e questões, e como lidar um com o outro. Para Jackson (2012, tradução nossa) o conhecimento reside nos traços de memória dos indivíduos, é o que eles sabem.

Dieng-Kuntz e Matta (2002, tradução nossa) ressaltam que a Memória Organizacional (MO) tem sido definida como uma abordagem da GC, na qual várias técnicas podem ser adotadas e são descritas como pertencentes a sua memória organizacional, que podem ser: o conhecimento individual, as aplicações referentes a tarefas de rotina, resolução de problemas e tomadas de decisão. Para Jackson (2012, tradução nossa) a MO existe numa variedade de formas e repositórios, como: documentos, bases de dados, cérebros dos empregados, e rituais do grupo. No entanto, utilizar de forma eficaz a MO é um dos maiores desafios para melhorar o escopo e disponibilidade dos estoques de conhecimento dentro das organizações. Para Walsh e Ungson (1991, tradução nossa) na medida em que as organizações exibem características de processamento de informação, incorporam algum tipo de memória, apesar de não necessariamente assemelhar-se à memória humana. No entanto, existem limitações para compreender os sistemas complexos que as organizações utilizam para armazenar conhecimento. Steil e Santos (2012, tradução nossa) afirmam que a contribuição do conhecimento organizacional tornou-se fundamental para a compreensão da memória organizacional, e estudos sobre aprendizagem organizacional tem contribuído para a memória organizacional, especialmente no que diz respeito à compreensão de como incorporam o conhecimento adquirido a partir da experiência em suas organizações. 
Tendo em vista a importância que o tema $\mathrm{MO}$ tem representado dentro das organizações e nas práticas de GC, esta pesquisa tem como objetivo mapear o termo Memória Organizacional, nas bases de publicações nacionais, das áreas de Administração, Ciências Contábeis e Turismo; Psicologia; e Interdisciplinar e também na Base de Dados do Evento EnANPAD. A construção desse conhecimento está representada nesta pesquisa pela seleção dos artigos relevantes que compõem o portfólio bibliográfico relacionado ao tema de MO, bem como a análise bibliométrica desses artigos. Sendo assim, na seção 2 o artigo apresenta os construtos sobre o tema trazidos pelos principais artigos das áreas investigadas. Na seção 3 apresenta o mapeamento científico e a técnica bibliométrica estabelecida. A seção 4 aponta os resultados da análise sistemática. E por fim, as conclusões e referências.

\section{MEMÓRIA ORGANIZACIONAL}

Walsh e Ungson (1991, tradução nossa) afirmam que, embora geralmente se reconheça que a MO consiste de artefatos mentais e estruturais, este assunto permanece fragmentado, não tendo sido ainda sintetizado em uma teoria consistente. Steil e Santos (2012, tradução nossa) reafirmam a existência de uma fragmentação na literatura sobre memória organizacional, juntamente com a necessidade de uma maior compreensão de como a aprendizagem e conhecimento são incorporados em organizações e como eles são recuperados. As "organizações são entidades mentais capazes de reflexão, de pensamento", mostrando, enfim, que essas e outras opiniões, situadas entre esses extremos, não são claras para instruir se a informação é estocada e processada pelas pessoas, as quais constituem a organização; pela organização em si ou pela coligação dominante ou escalão superior como um reflexo da organização. A MO é um construto em nível tanto individual quanto organizacional (WALSH e UNGSON, 1991, tradução nossa).

No campo organizacional, Chang, Choi e Lee (2004, tradução nossa) relatam que, um dos elementos que contém o conhecimento de que as organizações utilizam para gerenciar seus ativos é a MO, que é analisada por meio de uma variedade de áreas, tais como, bibliotecas digitais, bases de conhecimentos, documentos, apoio a decisão baseado em casos. Embora tais análises sejam praticadas, os autores afirmam que esclarecer como a MO utiliza o conhecimento organizacional, tanto explícito como tácito ainda é um tema em estudo.

Walsh e Ungson (1991, tradução nossa) acrescentam que a MO como construto é composto da estrutura da instalação de retenção, da informação nela contida, dos processos de aquisição e recuperação da informação e de suas consequências. Como conceito, afirmam que $\mathrm{MO}$ se refere à informação armazenada da história da organização, que pode ser trazida para auxiliar decisões atuais e esclarecem que as informações podem ser consideradas estímulos decisórios e respostas, que são preservadas em recipientes particulares de armazenagem, bins, que levam a consequências comportamentais quando recuperadas. E por fim, postulam que a MO pode estar retida em seis lugares, sendo 
cinco dentro da própria organização: indivíduos, cultura organizacional, transformações organizacionais, estruturas organizacionais e ecologia organizacional; e em um lugar fora da organização, que denominam de arquivos externos, sendo este qualquer elemento externo utilizado para resgatar ações e performances da própria organização. Na visão de Stein (1995, tradução nossa) a MO tem por definição seu conteúdo, considerando a soma de todo o conhecimento reunido pela organização e seus processos (codificação, armazenamento e aplicações, etc), sendo a forma de como o conhecimento é exercido sobre as atividades atuais da organização.

Em termos de trabalhos relacionados com a proposta desta pesquisa, Anderson e Sun (2010, tradução nossa) realizaram uma busca de revisão de literatura do termo MO na base de dados da Web of Science e Social Science Citation Index. O objetivo destes autores foi investigar o universo de pesquisadores que citaram o trabalho de J. Walsh e G. Ungson, no período de entre 1991 a 2006. Outro trabalho, relacionado com a proposta desta pesquisa, é o de Santos, Maldonado e dos Santos (2011) que teve por objetivo mapear o perfil das pesquisas acadêmico-científicas sobre MO realizada na base de dados da Web of Science e utilizaram a bibliometria como técnica de visualização e mapeamento científico. Este último artigo, em especial, faz parte do universo de pesquisa do presente trabalho, tendo sido publicado numa base de dados nacional. Todos os artigos citados nas pesquisas de Anderson e Sun (2010, tradução nossa) e Santos, Maldonado e Dos Santos (2011), fazem parte das bases internacionais, o que leva naturalmente à compreensão da importância da realização de uma revisão de literatura nas bases de dados nacionais que vise identificar, entre os trabalhos acadêmicos e científicos publicados, aqueles que tratam do conceito e da aplicação do termo MO. A próxima seção trata do mapeamento realizado para a presente pesquisa, nas bases de dados nacionais.

\section{MAPEAMENTO CIENTÍFICO - TÉCNICA BIBLIOMETRIA}

Tasca, et al. (2010, tradução nossa) salienta que o processo de pesquisa científica se inicia com um problema, pergunta ou dúvida, que motiva os pesquisadores a procurar informações sobre um dado tema em bibliotecas e bases bibliográficas digitais. Para Araújo, (2006), a bibliometria é uma técnica que permite situar a pesquisa através de diversos indicadores e diversas relações, uma vez determinada a área de conhecimento que será foco da pesquisa, evidencia-se a escolha das palavras-chave que serão utilizadas na busca de referências.

Segundo Santos e Kobashi (2009) é no século XX que esses métodos ganham densidade e legitimidade. A bibliometria, por sua vez, é muito utilizada e conhecida por pesquisadores da Ciência da Informação e tem como objetos de estudo os livros ou as revistas científicas, cujas análises se vinculam à gestão de bibliotecas e bases de dados. A análise de dados, para Santos e Kobashi (2009), constitui um elemento indispensável para fazer avançar a nossa compreensão sobre os estudos da ciência como um sistema complexo de produção e de troca de conhecimento. 
A bibliometria quando utilizada em bases de dados nacionais se difere em gênero, número e grau da realizada em bases internacionais. As bases internacionais apresentam filtros de inserção e exclusão de dados que facilitam as buscas. Já nas bases nacionais o processo requer uma investigação minuciosa do pesquisador, onde este deve ir montando sua própria base de dados manualmente. A seguir são apresentados os métodos utilizados para o desenvolvimento deste trabalho.

\subsection{Procedimentos metodológicos}

Nesta pesquisa, utilizou-se a técnica bibliometria na busca pelo termo Memória Organizacional nas bases de dados nacionais, das áreas Administração, Ciências Contábeis e Turismo; Psicologia; e Interdisciplinar com Qualis A1, A2, B1, B2 e B3, considerados mais relevantes e na base de dados do Encontro Nacional da Associação Nacional de Pós-Graduação e Pesquisa em Administração (EnANPAD), evento mais relevante da área da Administração, para investigar o conceito e aplicação deste termo nas pesquisas nacionais.

O processo de desenvolvimento deste estudo constituiu em duas etapas: busca sistemática e análise descritiva, que podem ser resumidas nos seguintes passos:

a) Identificação da base de dados - no Brasil a Capes é o órgão responsável pela certificação da produção intelectual dos programas de pós-graduação. $A$ estratificação da qualidade dessa produção é realizada de forma indireta. Dessa forma, o Qualis afere a qualidade dos periódicos e de outros tipos de produção, a partir da análise da qualidade dos veículos de divulgação, ou seja, periódicos científicos. A classificação de periódicos é realizada pelas áreas de avaliação e passa por processo anual de atualização. Esses veículos são enquadrados em estratos indicativos da qualidade - A1, o mais elevado; A2; B1; B2; B3; B4; B5 e C - com peso zero (COORDENAÇÃO DE APERFEIÇOAMENTO DE PESSOAL DE NÍVEL SUPERIOR, 2012).

b) Os critérios de seleção foram definidos pela pesquisa do Qualis Capes. Os periódicos nacionais selecionados estão enquadrados nos níveis $A 2, B 1, B 2$ e B3 das áreas Administração, Ciências Contábeis e Turismo; Psicologia; e Interdisciplinar. No nível A1, no período da pesquisa, a busca não apresentou periódicos nacionais. O corte da busca foi em B3, excluindo os níveis B4, B5 e $\mathrm{C}$ considerados pela Capes com peso baixo ou zero. Todos os periódicos selecionados na área de Administração tem o Qualis respectivo tanto para área de Psicologia como na área Interdisciplinar. Sendo a área da Administração a mais dotada de periódicos com o tema, decidiu-se realizar também uma busca na base de dados do EnANPAD, o evento da área de Administração mais relevante, realizando a busca nas bases de dados dos anos de 2001 a 2011 (último ano na data da realização da pesquisa). 
c) O resultado desta estratégia de busca na base Qualis Capes resultou em 388 periódicos, sendo que destes, 124 eram nacionais, nos níveis $A 2, B 1, B 2$ e B3. Do conjunto dos 124 periódicos nacionais foi realizada uma nova busca sistemática para o termo "memória" em cada periódico para aumentar o campo de busca, resultando em 56 periódicos com o termo memória.

d) Como o objetivo era mapear o termo Memória Organizacional e termos correlatos, como "memória coletiva" ou "memória de grupo", dos 56 periódicos somente 10 apresentaram tal informação. Na base do EnANPAD também foi realizada busca sistemática, entre os anos de publicação 2001 a 2011, em todos os volumes, usando o termo Memória Organizacional e seus correlatos, onde foram encontrados 9 artigos.

e) As análises dos artigos selecionados foram feitas a partir da elaboração de tabelas e gráficos sobre os principais dados bibliométricos, tais como: dados de referências, de autores, número de citações e periódicos mais relevantes e tipos de pesquisa utilizada nos artigos selecionados, tais como estudo de caso ou revisão de literatura. A análise do tipo de pesquisa revisão de literatura poderia trazer ao trabalho contribuições a respeito de um construto sobre 0 termo MO extraído das bases nacionais.

Na próxima seção apresentam-se os resultados mais relevantes desta pesquisa levando em consideração que o termo Memória Organizacional refere-se a um tema relativamente novo nas bases de busca nacionais e pouco explorado como tema de pesquisa científica.

\section{RESULTADOS}

No presente estudo, ao mapear o termo Memória Organizacional (MO), exclusivamente em bases de publicações nacionais relevantes, pesquisadas e analisadas conforme indicado nas seções a seguir, somente em um dos artigos de toda a pesquisa foi encontrada, nas suas considerações finais, o que poderia ser entendido como sendo uma definição sobre o que seja a área de MO: "investigar e auxiliar a compreensão de como as organizações recuperam, acessam, armazenam, usam e reusam conhecimentos experienciais gerados ao longo do tempo, para gerar vantagem competitiva"(SANTOS, MALDONADO, DOS SANTOS, 2011). Neste artigo citado, bem como nos demais artigos da presente pesquisa, observa-se que as definições de $\mathrm{MO}$ neles utilizadas fazem parte das bases internacionais, ou seja, em termos de publicações nacionais relevantes ainda não há uma definição específica para o termo Memória Organizacional, apenas a indicação aqui mencionada. Na próxima seção será abordada a bibliometria como técnica de mapeamento científico, o que fornece o suporte metodológico para esta pesquisa. 


\subsection{Resultados da busca sistemática de literatura}

De acordo com a análise bibliométrica realizada, foram localizados 10 artigos indexados nos periódicos nacionais com Qualis Capes entre A2 e B3, no período de outubro de 2012, mais 9 artigos publicados no EnANPAD nos anos de 2000 a 2011. Em razão de ter ocorrido uma duplicidade de publicação em 2 artigos do EnANPAD, publicados posteriormente em outros periódicos nacionais, o número total de artigos contabilizados do EnANPAD passou a ser 7. Os 17 trabalhos selecionados foram escritos por 48 autores de 19 instituições diferentes. Para escrever estes artigos seus autores utilizaram 492 referências, o que dá uma média de 28 referências por artigo. E publicados em 11 fontes diferentes, (10 periódicos e 1 evento). A Tabela 1 apresenta os dados gerais da pesquisa.

Tabela 1: Resultado geral do levantamento bibliométrico

\begin{tabular}{l|c}
\hline \multicolumn{1}{c|}{ CRITÉRIOS } & FREQUÊNCIA \\
\hline Publicações & 17 \\
\hline Fontes de publicação & 11 \\
\hline Autores & 48 \\
\hline Instituições & 19 \\
\hline Referências Citadas & 492 \\
\hline Palavras-chave informadas & 31 \\
\hline Palavras-chave sugeridas & 20 \\
\hline
\end{tabular}

Fonte: Dos autores

Em relação às publicações, notou-se que no Brasil, o estudo sobre o tema Memória Organizacional é relativamente novo, passando a ter publicações de fato a partir de 2004, já que o artigo de 1982 aborda o tema memória no sentido de pesquisa histórica. A Tabela 2 apresenta esta frequência de publicações sobre o tema e estão relacionados com estudos de aprendizagem organizacional e gestão do conhecimento, conforme as palavras-chaves informadas nos artigos observados.

Tabela 2: Frequência de publicações por ano

\begin{tabular}{c|c}
\hline ANO & QUANTIDADE \\
1982 & 1 \\
2004 & 2 \\
2005 & 1 \\
2006 & 3 \\
2007 & 1 \\
2009 & 4 \\
2010 & 3 \\
2011 & 2 \\
TOTAL & 17 \\
\hline
\end{tabular}

Fonte: Dos autores 
O artigo de 1982 tem como foco práticas organizacionais e memória coletiva, onde os autores levantam dados sociais das práticas organizacionais dominantes na lugoslávia agrária, procurando compreender a aparição da autogestão no país no início da década de 1980, por meio da memória coletiva, que aqui é compreendida como um conjunto de conhecimentos institucionalizados ou enraizados sobre a realidade das comunidades domésticas do campo até a década de 1950. A partir desta data de publicação temos uma lacuna no tempo voltando a ter publicações do termo MO somente a partir de 2004 com foco nas organizações, seguindo uma linha contínua, com exceção do ano de 2008.

A Tabela 3, a seguir, apresenta os periódicos nacionais selecionados na busca sistemática onde foram encontrados os 10 artigos e seus respetivos Qualis/Capes, visualizados no período de Agosto a Outubro de 2012, nas áreas Administração, Ciências Contábeis e Turismo (ADM), Psicologia (PSC) e Interdisciplinar (INTER).

Tabela 3: Periódicos e Qualis/Capes respectivo*

\begin{tabular}{cl|c|c|c|c}
\hline PERIÓDICO & $\begin{array}{l}\text { Qtd. } \\
\text { Artigos }\end{array}$ & $\begin{array}{c}\text { Qualis } \\
\text { ADM }\end{array}$ & $\begin{array}{c}\text { Qualis } \\
\text { PSC }\end{array}$ & Qualis INTER \\
O\&S & 1 & A2 & B3 & B2 \\
RAC & 1 & A2 & - & - \\
RAE & 1 & A2 & B1 & B1 \\
Análise PUCRS Online & 1 & B2 & - & - \\
Ciência e Cognição & 1 & B2 & B3 & B3 \\
Perspectivas Contemporâneas & 1 & B2 & - & B4 \\
RAM & 1 & B1 & B1 & - \\
RAUSP & 1 & B1 & B3 & B2 \\
RBGN & 1 & B1 & - & B3 \\
Datagramazero & 1 & B3 & B4 & B3 \\
TOTAL & 10 & & & \\
\hline
\end{tabular}

Fonte: Coordenação de Aperfeiçoamento de Pessoal de Nível Superior (2012)

A Tabela 4 apresenta as análises das buscas sistemáticas iniciais para a coleta final dos 17 artigos com o termo $\mathrm{MO}$, onde destes, 10 foram resultantes de periódicos nacionais e 7 da base de dados do EnANPAD, compreendendo os períodos de 2001 a 2011. Para a seleção dos periódicos da base de dados da Capes foram analisados periódicos com Qualis A1, A2, B1, B2 e B3 das áreas de Administração, Ciências Contábeis e Turismo (ADM), Psicologia (PSC) e Interdisciplinar (INTER) eliminando os periódicos que se repetiam entre as áreas. Para o Qualis $A 1$, por exemplo, dos 76 periódicos que a busca apresentou, no site do <qualis.capes.gov.br>, não foram encontrados periódicos 
nacionais no período da busca. Já para o Qualis $A 2$, dos 82 periódicos que a busca apresentou, 14 eram periódicos nacionais e assim sucessivamente para o restante dos Qualis. Dos 464 artigos totais visualizados no site, apenas 124 periódicos eram nacionais. A partir destes dados foi elaborada uma nova pesquisa, em cada periódico, com o termo genérico "memória", resultando em 56 periódicos. E por fim, uma nova pesquisa com o termo "memória organizacional" resultando em 10 periódicos nacionais, contendo um artigo por periódico sobre o tema, conforme apresentado a seguir.

Tabela 4: Busca sistemática de periódicos

\begin{tabular}{c|c|l|c|l}
\hline & N. Periódicos & $\begin{array}{l}\text { N. } \\
\text { Periódicos } \\
\text { Nacionais }\end{array}$ & $\begin{array}{l}\text { N. Periódicos c/ } \\
\text { termo Memória }\end{array}$ & $\begin{array}{l}\text { Periódicos } \\
\text { c/ termo } \\
\text { Mo }\end{array}$ \\
A1 & 76 & - & - & - \\
A2 & 82 & 14 & 9 & 3 \\
B1 & 102 & 27 & 32 & 3 \\
B2 & 52 & 46 & 7 & 3 \\
B3 & 152 & 37 & 8 & 1 \\
TOTAL & 464 & 124 & 56 & 10 \\
\hline
\end{tabular}

Fonte: Dos autores

Na Tabela 5, o mesmo procedimento de busca foi realizado para as Bases de Dados anuais do EnANPAD, considerado o maior evento da área da Administração, compreendendo os períodos de 2001 a 2011. Primeiramente a busca em cada base/ano de publicação foi realizada apenas com o termo "memória" encontrando 18 artigos. Destes analisou-se cada um para verificar artigos que tratassem apenas sobre "memória organizacional" ou correlatos, restando 9 artigos. Foram excluídos 2 artigos desta seleção do EnANPAD um do ano de 2006 e outro publicado em 2007, pois estes foram publicados nos periódicos da Tabela 3 respectivamente, em 2007 na revista RBGN e em 2009 na revista RAM. 
Tabela 5: Busca Sistemática na Base de Dados EnANPAD

\begin{tabular}{c|c|c}
\hline ANO & N. Artigos c/ termo Memória & N. Artigos c/ termo MO \\
2001 & - & - \\
2002 & - & - \\
2003 & - & - \\
2004 & 4 & 2 \\
2005 & 1 & - \\
2006 & 2 & $2(-1)$ \\
2007 & 2 & $1(-1)$ \\
2008 & - & - \\
2009 & 1 & 1 \\
2010 & 4 & 2 \\
2011 & 4 & 1 \\
TOTAL & 18 & 9 \\
\hline
\end{tabular}

Quanto aos autores e respectivas instituições, dos 17 artigos selecionados, a Tabela 6 mostra um total de 19 instituições distribuídas em 07 Estados brasileiros. O Estado com maior número de publicação é SP seguido do RS em segundo. SC é o estado que apresenta maior número de autores por artigo.

Tabela 6: Dados Autores e Instituições

\begin{tabular}{c|c|c}
\hline INSTITUIÇÃO & AUTORES & UF \\
FEA/USP & 3 & SP \\
FECAP & 1 & SP \\
FFLCH & 1 & SP \\
FGV & SP \\
FIA & 3 & SP \\
MACKENZIE & 1 & SP \\
UNIFEI & 1 & SP \\
USP & 1 & SP \\
PUC & 2 & RS \\
UFRGS & 2 & RS \\
UNISINOS & 4 & RS \\
FURB & 3 & SC \\
UFSC & 7 & SC \\
UFMG & 9 & MG \\
UFPE & 2 & PE \\
UFRJ & 1 & RJ \\
UNB & 1 & DF \\
PUC/PerU & 5 & \\
Total 19 & 1 & $\mathbf{7}$ \\
\hline
\end{tabular}

Fonte: Dos autores 
A seguir, a Tabela 7 apresenta de forma detalhada os resultados das referências utilizadas na construção dos 17 artigos selecionados. Foram utilizadas para construção dos 17 artigos 492 obras, escritas por 809 autores. Destas obras 319 são internacionais e 173 nacionais e ainda o tipo de obra sendo que as mais utilizadas são Artigos (231) e Livros (226).

Tabela 7: Detalhamento das obras utilizadas na construção dos artigos selecionados

\begin{tabular}{|c|c|c|}
\hline \multicolumn{3}{|c|}{ Total de artigos localizados=17 ARTIGOS } \\
\hline \multicolumn{2}{|c|}{492 REFERÊNCIAS } & 809 AUTORES \\
\hline 319 Internacionais & 173 Nacionais & \\
\hline TIPO DE OBRA & QTD & \\
\hline Anais & 2 & \\
\hline Artigo & 231 & \\
\hline Ata & 3 & \\
\hline Dicionário & 1 & \\
\hline Dissertação & 3 & \\
\hline Enciclopédia & 2 & \\
\hline Excel & 1 & \\
\hline Jornal & 1 & \\
\hline Lei/Resolução & 2 & \\
\hline Livro & 226 & \\
\hline Mimeógrafo & 1 & \\
\hline Relatório & 6 & \\
\hline Site & 7 & \\
\hline Tese & 6 & \\
\hline
\end{tabular}

A Tabela 8 apresenta uma relação de obras internacionais e nacionais utilizadas na construção dos 17 artigos selecionados, contendo o termo Memória Organizacional em seu título e que foram citadas mais de uma vez. Entretanto, não foram identificadas referências nacionais, dentre as referências, sobre MO utilizadas pelos 17 trabalhos analisados. 
Tabela 8: Obras/referências utilizadas com o termo MO

\begin{tabular}{l|c|c}
\hline \multicolumn{1}{c|}{ REFERÊNCIA COMPLETA } & QTD & TIPO \\
\hline Organizational memory & 5 & Artigo \\
\hline Actualizing organizational memory with information systems & 2 & Artigo \\
\hline $\begin{array}{l}\text { Designing Organizational Memory: Preserving Intellectual } \\
\text { Exploring the Impacts of Knowledge (Re)Use and Organizational } \\
\text { Memory on the Effectiveness of Strategic Decisions: A Longitudinal } \\
\text { Case Study }\end{array}$ & 2 & Artigo \\
$\begin{array}{l}\text { How Can Organizational Memory Theories Contribute to } \\
\text { Organizational Memory Systems? }\end{array}$ & 2 & Artigo \\
$\begin{array}{l}\text { Organizational memory information systems: a transactive memory } \\
\text { approach }\end{array}$ & 2 & Artigo \\
$\begin{array}{l}\text { Organizational memory systems: challenges for information } \\
\text { technology }\end{array}$ & 2 & Artigo \\
$\begin{array}{l}\text { Reexamining organizational memory } \\
\text { The impact of organizational memory on new product performance } \\
\text { and creativity }\end{array}$ & 2 & Artigo \\
\hline \begin{tabular}{l} 
Toward a technology for organizational memories \\
\hline
\end{tabular} & 2 & Artigo \\
\hline
\end{tabular}

Fonte: Dos autores

De igual forma a Tabela 9 apresenta uma relação dos 10 autores, com suas respectivas obras, mais citados do total de 492 referências nos 17 artigos selecionados. Nesta tabela é possível observar que as obras utilizadas que abordam o tema MO são todas do tipo artigos, cuja autoria de: ABEL, VASCONCELLOS e KIMBLE; WALSH e UNGSON; e ACKERMAN. Na área de Gestão do Conhecimento os autores mais utilizados são NONAKA e TAKEUCHI, e DAVENPORT. E ARGYRIS e SCHÖN os autores mais citados sobre aprendizagem organizacional. 
Tabela 9: Análise das referências dos artigos nacionais sobre MO: os 10 Autores mais citados e as suas obras

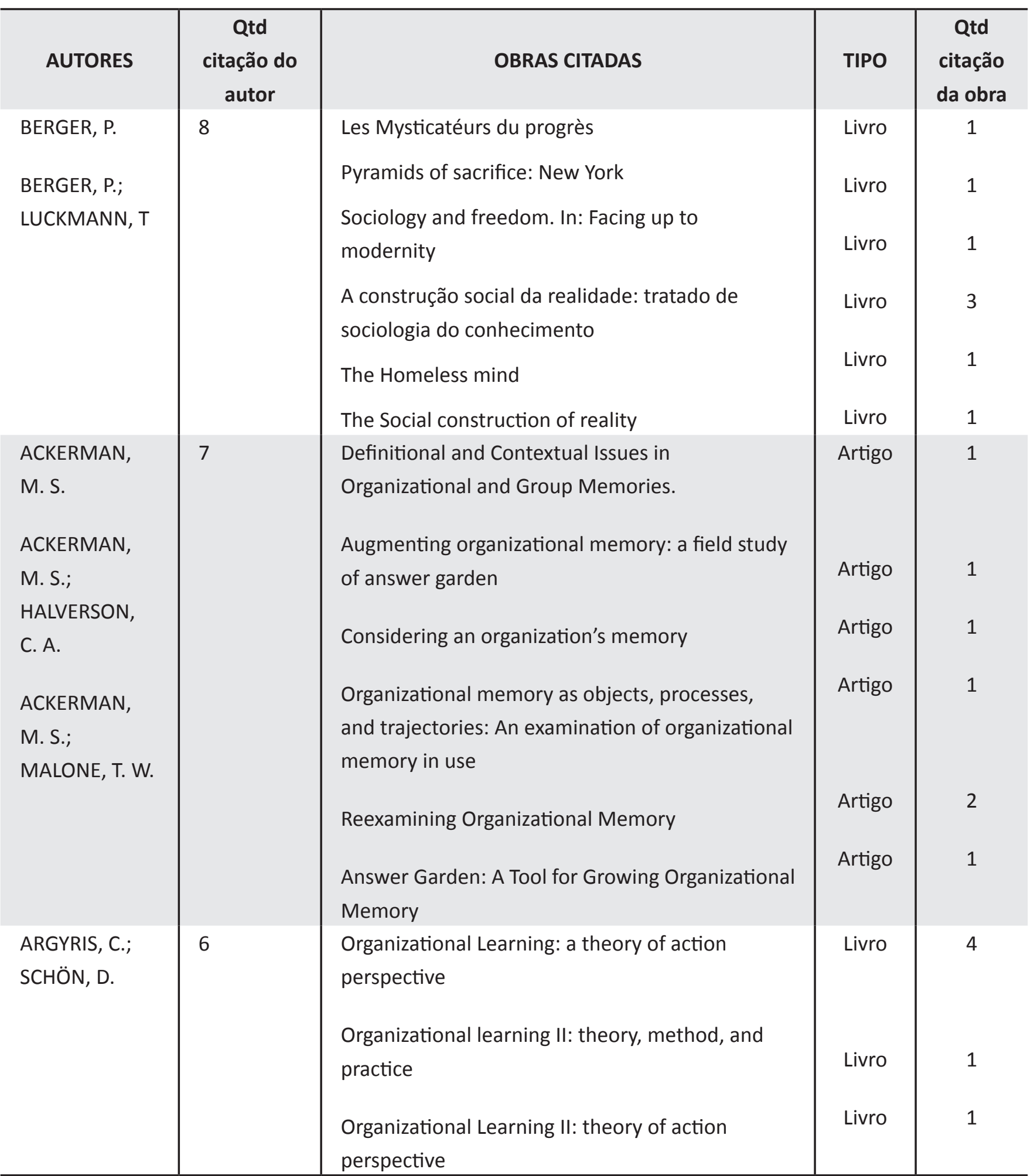




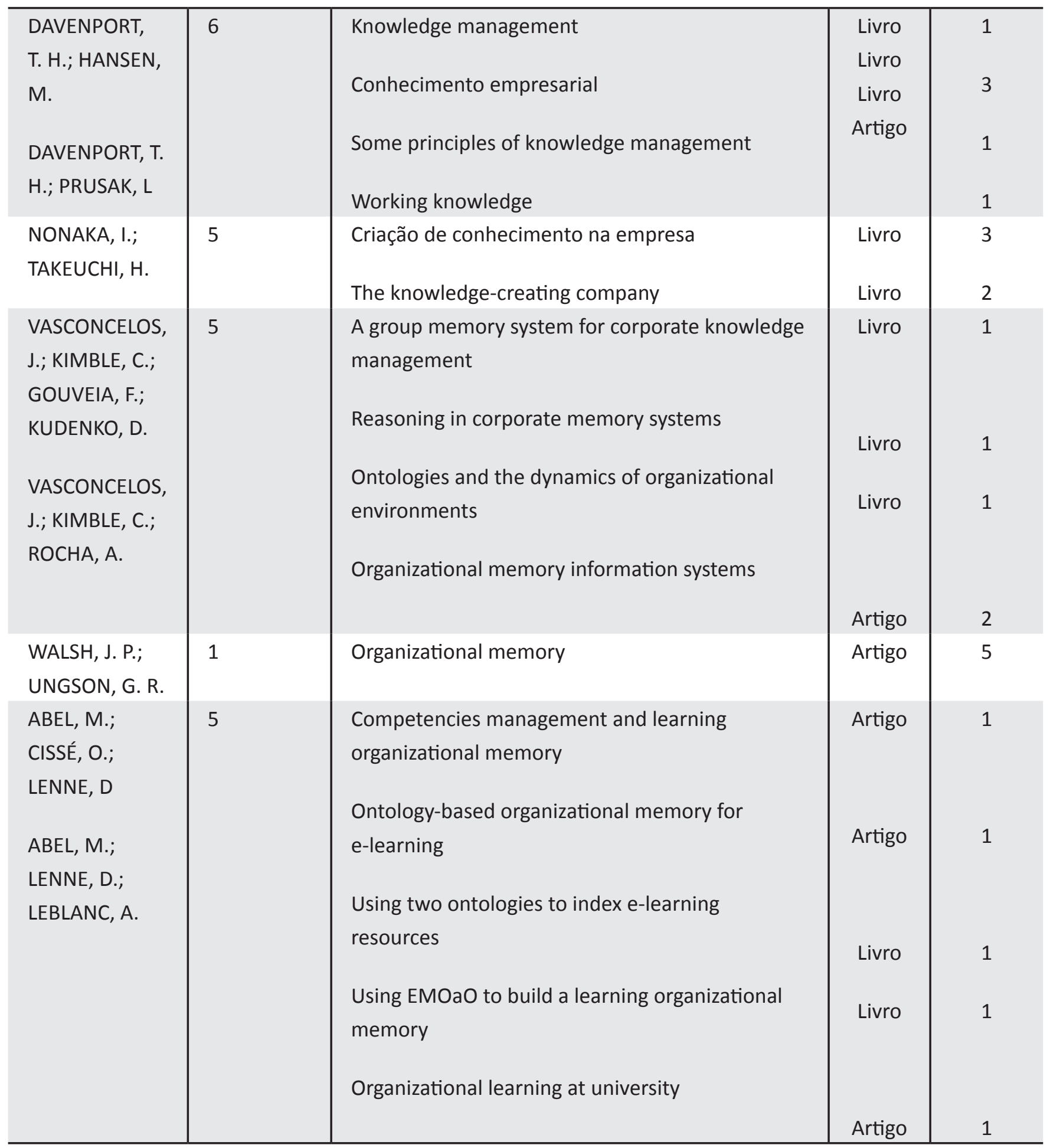




\begin{tabular}{l|l|l|c|c}
\hline $\begin{array}{l}\text { FLEURY, A.; } \\
\text { FLEURY, M. }\end{array}$ & 5 & Aprendizagem e inovação organizacional & Livro & 1 \\
T. L. & & $\begin{array}{l}\text { Estratégias empresariais e formação de } \\
\text { competências }\end{array}$ & Livro & Artigo \\
FLEURY, M. T. & & Construindo o conceito de competência & Livro \\
L.; OLIVEIRA \\
JR., M.
\end{tabular}

Fonte: Dos autores

Em se tratando de conceito de MO informada nos artigos selecionados, destaca-se a citação encontrada no artigo publicado na revista RAUSP em 2005, de Walsh e Ungson (1991, tradução nossa) da obra Organizational Memory, que definem MO como informação passada que pode ser utilizada no presente para a tomada de decisão, sendo composta por três categorias conceituais: conteúdo; estrutura e processos de aquisição; estocagem e recuperação. Outra citação, dos mesmos autores, encontrada no artigo publicado no EnANPAD de 2011, informa que "as interpretações do passado podem ser incorporadas nos sistemas e artefatos, por exemplo, as estruturas, as transformações, a ecologia".

Outro exemplo é de um artigo publicado no EnANPAD de 2006 que complementa o conceito de MO apresentando o processo de MO, adaptado de Walsh e Ungson (1991, tradução nossa) e outros autores, porém esses não foram os mais citados conforme a Tabela 9. O processo é representado pelo fluxo da informação, desde sua aquisição, passando pela retenção num Repositório ou Base de MO (sistema informatizado), que ao ser acessado, o usuário poderá buscar, recuperar, reutilizar ou descartar informações. Em tal modelo proposto, a Base inclui não só o recurso de banco de dados provido pela tecnologia da informação, mas também o conhecimento explícito (ou informação) contido nos meios físicos (normas, manuais, documentos, vídeos), bem como os meios que armazenam o conhecimento tácito. 


\subsection{Resultados da análise descritiva dos artigos selecionados}

O objetivo aqui é apresentar qual o tipo de pesquisa foi definida nos 17 artigos selecionados, bem como categorizar em grupos de acordo com o método de abordagem definido nos artigos. Destaca-se na Tabela 10 outra categorização realizada nos 17 artigos selecionados onde foi possível verificar e dividir em dois grupos de análise - o tipo de pesquisa e a abordagem metodológica. Dos 17 artigos, 10 artigos são pesquisas empíricas de Estudo de Caso; 5 artigos são Revisão de Literatura; e os 2 artigos restantes apresentam outros métodos. Para esta pesquisa interessa os artigos de Revisão de Literatura que podem contribuir para a construção de um construto nacional do termo MO e os de Estudo de Caso, que podem contribuir para uma análise de como a MO é percebida nas organizações brasileiras.

Tabela 10: Classificação dos artigos por Tipo de Pesquisa e Método de Abordagem

\begin{tabular}{|l|l|l|l}
\hline ANO PUBL. & QTD. PUBL. & TIPO PESQUISA & MÉTODO DE ABORDAGEM \\
\hline 1982 & 1 & Empírico & Estudo de Caso \\
\hline 2004 & 2 & Empírico & Estudo de Caso \\
2005 & 1 & Não Empírico & Revisão de Literatura \\
\hline 2006 & 2 & Empírico & Estudo de Caso \\
\hline 2006 & 1 & Não Empírico & Revisão de Literatura \\
\hline 2007 & 1 & Empírico & Estudo de Caso \\
\hline 2009 & 1 & Não Empírico & Revisão de Literatura \\
\hline 2009 & 1 & Empírico & Quali/Quanti \\
\hline 2009 & 2 & Empírico & Estudo de Caso \\
\hline 2010 & 2 & Empírico & Estudo de Caso \\
\hline 2010 & 1 & Não Empírico & Revisão de Literatura \\
\hline 2011 & 1 & Não Empírico & Revisão de Literatura \\
\hline 2011 & 1 & Não Empírico & Modelo Conceitual \\
\hline TOTAL & 17 & & \\
\hline
\end{tabular}

Fonte: Dos autores

A Tabela 11, a seguir, apresenta os artigos selecionados com abordagem na Revisão de Literatura analisando cada um em particular. 
Tabela 11: Artigos com abordagem Revisão de Literatura

\begin{tabular}{|c|c|c|c|c|c|}
\hline N. & TíTULO & N. autores & N. Ref. & Pub. & ANO \\
\hline 1 & $\begin{array}{l}\text { Um Checklist para } \\
\text { avaliação de requisitos } \\
\text { de memória de } \\
\text { trabalho no Nível } 2 \text { do } \\
\text { modelo P-CMM }\end{array}$ & 12 & 13 & Ciência e Cognição & 2006 \\
\hline 2 & $\begin{array}{l}\text { Aprendizagem } \\
\text { organizacional: uma } \\
\text { análise sobre o } \\
\text { debate e a escolha } \\
\text { de categorias para } \\
\text { estudos de caso }\end{array}$ & 2 & 27 & $\begin{array}{c}\text { Perspectivas } \\
\text { Contemporâneas }\end{array}$ & 2009 \\
\hline 3 & $\begin{array}{l}\text { O que Lembrar e o que } \\
\text { Esquecer? Memória } \\
\text { e a Formalização } \\
\text { Social do Passado nas } \\
\text { Organizações }\end{array}$ & 2 & 30 & EnANPAD & 2010 \\
\hline 4 & $\begin{array}{l}\text { Utilizando estoques } \\
\text { de conhecimento } \\
\text { organizacional: um } \\
\text { quadro de referência }\end{array}$ & 2 & 32 & RAUSP & 2005 \\
\hline 5 & $\begin{array}{l}\text { Mapeamento } \\
\text { das Publicações } \\
\text { Acadêmico-Científicas } \\
\text { sobre Memória } \\
\text { Organizacional }\end{array}$ & 3 & 33 & EnANPAD & 2011 \\
\hline
\end{tabular}

Fonte: Dos autores

A partir da leitura dos textos completos dos artigos, de abordagem metodológica revisão de literatura, observa-se que no Brasil, pesquisas sobre $\mathrm{MO}$ ainda se mostram como um campo em desenvolvimento. A temática é desafiadora e fortemente vista como um quadro histórico empresarial e cultural (Conforme artigos 1, 2 e 3, Tab.11). No entanto, estudos sobre Aprendizagem Organizacional e Gestão do Conhecimento têm levantando algumas discussões sobre a importância da $\mathrm{MO}$, onde estoques de conhecimento são resultantes de aprendizagem e aprimoramento da capacidade competitiva de uma organização (Conforme artigo 4). 
Com a intenção de analisar a forma como as organizações recuperam e armazenam conhecimentos (conforme artigo 5), a MO pode ser definida como uma importante ferramenta organizacional para investigar e auxiliar na compreensão em termos de seu conteúdo (conhecimento) e dos processos associados, tais como - aquisição, codificação, armazenamento, comunicação, utilização, recuperação, etc., gerados ao longo do tempo, para gerar vantagem competitiva e garantir sobrevivência das organizações.

Tabela 12: Artigos com abordagem Estudo de Caso

\begin{tabular}{|c|c|c|c|c|c|}
\hline N. & TíTULO & N. autores & N. Ref. & Pub. & ANO \\
\hline 1 & $\begin{array}{l}\text { A institucionalização de tipologias } \\
\text { organizacionais. Um estudo de caso: a } \\
\text { autogestão na lugoslávia }\end{array}$ & 1 & 26 & RAE & 1982 \\
\hline 2 & $\begin{array}{l}\text { Os Programas De Melhoria Contínua Como } \\
\text { Processos De Aprendizagem Organizacional: O } \\
\text { Caso De Uma Indústria De Produtos Alimentícios }\end{array}$ & 2 & 17 & EnANPAD & 2004 \\
\hline 3 & $\begin{array}{l}\text { As Percepções Subjetivas do Tempo nas } \\
\text { Organizações e a Mudança Organizacional: Uma } \\
\text { Análise Comparativa da Daimler Chrysler e da } \\
\text { Bull }\end{array}$ & 3 & 36 & EnANPAD & 2004 \\
\hline 4 & $\begin{array}{l}\text { Organizational memory and forgetfulness } \\
\text { generating vulnerabilities in complex } \\
\text { environments }\end{array}$ & 1 & 28 & RAC & 2006 \\
\hline 5 & $\begin{array}{l}\text { Aprendizagem e Criação do Conhecimento em } \\
\text { Incubadoras }\end{array}$ & 3 & 21 & EnANPAD & 2006 \\
\hline 6 & $\begin{array}{l}\text { Memória Organizacional e reutilização do } \\
\text { conhecimento técnico em uma empresa do setor } \\
\text { eletrônico do Brasil }\end{array}$ & 2 & 40 & RBGN & 2007 \\
\hline 7 & $\begin{array}{l}\text { Conselho Tutelar: Competências e Processo de } \\
\text { Escolha de Conselheiros }\end{array}$ & 1 & 24 & EnANPAD & 2009 \\
\hline 8 & $\begin{array}{l}\text { Memória Organizacional de Comunidades de } \\
\text { Prática como Fator de Vantagem Competitiva das } \\
\text { Organizações }\end{array}$ & 4 & 28 & O\&S & 2009 \\
\hline 9 & $\begin{array}{l}\text { A Memória Institucional como Possibilidade de } \\
\text { Comunicação Organizacional: o caso do Exército } \\
\text { Brasileiro }\end{array}$ & 2 & 16 & $\begin{array}{l}\text { Análise } \\
\text { PUCRS } \\
\text { Online }\end{array}$ & 2010 \\
\hline 10 & $\begin{array}{l}\text { Aprendizagem Organizacional a partir das Práticas } \\
\text { de Educação a Distância da EA/UFRGS }\end{array}$ & 4 & 24 & EnANPAD & 2010 \\
\hline
\end{tabular}

Fonte: Dos autores 
Da mesma forma, os artigos da Tabela 12, com abordagem metodológica estudo de caso foram analisados para identificar como a MO é percebida e descrita nas organizações. Os artigos 1, 3 e 7 utilizam o termo memória como uma referência histórica, tanto social como organizacional e da importância de sua preservação em processos de mudança, e de como os atores envolvidos reconstroem o sentido de suas experiências a partir das transformações que vivenciam em seu cotidiano.

Os artigos 2, 5 e 10 abordam o tema aprendizagem organizacional como uma ferramenta facilitadora nos processos de mudança e criação de conhecimento. A memória organizacional é tratada no artigo 2 especificamente como uma dimensão de análise que caracteriza a aprendizagem organizacional. No artigo 10, a MO é uma das categorias analisadas no contexto das práticas de Educação a Distância (EaD) apresentando como o conhecimento é obtido, registrado e armazenado a partir das diferentes experiências, em sistemas de informação, constituindo um histórico a respeito da EaD na instituição em questão.

Quanto aos artigos 4, 6, 8 e 9 versam sobre o tema MO mais especificamente. No artigo 4 é feita uma análise entre $\mathrm{MO}$ e esquecimento onde $\mathrm{MO}$ é o poder ou o processo de reprodução ou de recuperação do que foi aprendido ou retido, principalmente através de mecanismos associativos, utilizando sistemas de informação para armazenar as experiências individuais e tem objetivo de reduzir custos de transação, auxiliar na velocidade de acesso a práticas do passado e soluções, ajudar na tomada de decisões e na redução da dependência da organização em relação aos indivíduos. Se essa memória for facilmente perdida ou quase inútil, não vai ajudar a organização a buscar vantagens competitivas, desenvolver a aprendizagem organizacional. No artigo 6 investiga práticas voltadas a formação de uma MO. Tais práticas estão atreladas a um repositório ou base de sistema informatizado que intermedia os processos de aquisição e retenção de informações e possibilita a busca/recuperação, (re) utilização ou descarte dessas informações pelos usuários.

O artigo 8 apresenta um estudo sobre Comunidades de Prática (CoPs) como um fator competitivo e seu papel na GC e MO observando uma organização que cria e compartilha conhecimento por meio dos inter-relacionamentos de aprendizagem dos seus integrantes. Explica que a MO das CoPs não é composta somente de documentos e listas de discussões mas também de rotinas, processos, práticas e normas organizacionais que auxiliem na construção de novos conhecimentos e aprendizagens individuais e organizacionais. E no artigo 9 a MO é chamada de Memória Institucional (MI) e tem como propósito facilitar no processo de comunicação organizacional e (re)construir relacionamentos de valor entre organização e seu meio complexo social. A MI é vista como um lugar, no qual o público interno pode se manter coeso e onde possam ser observados os aspectos do percurso organizacional que foram significativos e que podem ser reutilizados no presente organizacional.

Também foram analisados os dois artigos restantes, de abordagem quali/quanti e modelo conceitual conforme a Tabela 13. 
Tabela 13: Artigos com outras abordagens

\begin{tabular}{l|l|l|l|l|l}
\hline N. & TítULO & N. autores & N. Ref. & Pub. & ANO \\
\hline 1 & $\begin{array}{l}\text { Mecanismos de aprendizagem em } \\
\text { organizações: desenvolvimento e } \\
\text { validação de uma escala de medida }\end{array}$ & 1 & 21 & $\begin{array}{l}\text { RAM } \\
\text { Quali/quanti }\end{array}$ & 2009 \\
$\begin{array}{l}\text { A Memória Organizacional no } \\
\text { Contexto da Engenharia do } \\
\text { Conhecimento }\end{array}$ & 3 & 76 & $\begin{array}{l}\text { Datagramazero } \\
\text { Modelo conceitual }\end{array}$ & 2011 \\
\hline
\end{tabular}

Fonte: Dos autores

O artigo 1 é um estudo sobre Aprendizagem Organizacional, onde o autor procura validar uma Escala de Mecanismos de Aprendizagem em Organizações (Emao), a partir da Measurement Scale of Organizational Learning de López, Peón e Ordás (2005), a qual reconhece o caráter multidimensional da aprendizagem e coleta informações explícitas acerca de quatro dimensões teoricamente identificadas: aquisição, distribuição, interpretação e memória organizacional. Ou seja, apesar do artigo apresentar o termo MO na busca, aqui é apenas uma dimensão de análise que se refere à estocagem de conhecimentos em sistemas informatizados, regras, procedimentos, entre outros sistemas, para serem utilizados no futuro.

Neste sentido, o artigo 2, procura elucidar trazendo uma definição de memória organizacional enquanto um sistema que armazena as experiências vividas da organização. Os autores analisam métodos e técnicas da Engenharia do Conhecimento (EC) para descrever a $\mathrm{MO}$ e que possam dar suporte aos seus processos. A Engenharia do Conhecimento se foca na aplicação de metodologias e tecnologias que permitem adquirir, estruturar, formalizar e operacionalizar informações e conhecimentos existentes em domínios de problemas intensivos em conhecimento. A memória organizacional preocupa-se com a reutilização e compartilhamento do conhecimento armazenado nela, devido a isto, os sistemas de informação de memória organizacional geralmente encontram-se organizados em torno a três processos: aquisição, organização e recuperação do conhecimento.

Percebe-se que pesquisas em memória organizacional no Brasil ainda estão incubadas e restritas a casos específicos, porém relacionadas aos temas sobre gestão do conhecimento organizacional, aprendizagem organizacional e sistemas de informação. 


\section{CONCLUSÕES}

Este estudo realizou um mapeamento do termo Memória Organizacional nas bases de dados nacionais, levantando artigos científicos de relevância nos periódicos das áreas de Administração, Ciências Contábeis e Turismo; Psicologia; Interdisciplinar; e da base do EnANPAD. A análise dos artigos selecionados foi realizada por meio de uma busca sistemática, aplicando-se técnicas bibliométricas que permitiram observar de forma quantitativa e qualitativa as informações e conhecimentos científicos no campo da Memória Organizacional no âmbito nacional.

Foi possível investigar de que forma as pesquisas nacionais tem abordado o tema Memória Organizacional tanto em artigos de revisão de literatura quanto em estudo de caso. No que se refere à revisão de literatura os artigos mostraram, como construto, que a MO é definida como um sistema que armazena as experiências vividas na organização; ou ainda uma ferramenta organizacional para investigar e auxiliar na compreensão em termos de seu conteúdo (conhecimento) e dos processos associados, tais como aquisição, codificação, armazenamento, comunicação, utilização, recuperação que possibilite ao longo do tempo gerar vantagem competitiva e garantir sobrevivência das organizações. Neste sentido, percebe-se que para a idealização deste construto é necessário um arcabouço tecnológico organizacional que permita práticas e processos de MO.

Já no sentido de aplicação, a MO é descrita nos estudos de casos científicos, onde se percebeu que a memória pode ser compreendida por um marco, ou seja, o percurso histórico social e cultural que uma organização passa em seu ciclo de vida, e que esta memória pode ser resgatada tanto para melhorar seus processos como para fortalecer sua imagem. Não obstante, as práticas descritas estão atreladas a um repositório ou base de sistema informatizado que intermedia os processos de aquisição e retenção de informações e possibilita a busca/recuperação, (re)utilização ou descarte dessas informações pelos usuários, caracterizando novas aprendizagens.

A pesquisa revelou ainda que estudos sobre o tema são relativamente novos no Brasil, datados a partir do ano de 2004, com exceção do artigo de 1982, o qual tratou do termo mais como memória coletiva da organização social do país em questão. Nos demais temas, Memória Organizacional aparece associada com o termo Aprendizagem Organizacional e nos artigos mais recentes com o tema Gestão do Conhecimento (GC) e Engenharia do Conhecimento (EC).

Este estudo mostrou que o campo de estudo em questão abre novas oportunidades de pesquisas empíricas e não empíricas para a gestão do conhecimento organizacional, pois o termo memória está relacionado com pesquisas tanto na GC como EC. Dentro das pesquisas empíricas se destacaram neste trabalho, o estudo de caso e a pesquisa quali/quanti. Estas práticas são relevantes por integrar outras áreas do conhecimento, que permitem analisar uma realidade para compor um conceito. Auxiliam também na integração do processo cognitivo da atividade acadêmica, a qual promove um debate teórico que possa contribuir para elaboração de novos construtos e aplicações nacionais. 


\title{
ORGANIZATIONAL MEMORY: A BIBLIOMETRIC REVIEW ON A NATIONAL BASIS
}

\begin{abstract}
Given the importance that the topic Organizational Memory (OM) has assumed within organizations and practices of knowledge management, this research aims to map the term MO, on the basis of national publications in the areas of Administration, Accounting and Tourism, Psychology, Interdisciplinary and also in database of the EnANPAD event. The bibliometric technique was used in systematic search for the term $\mathrm{MO}$ in national databases in journals with Qualis A1, A2, B1, B2 and B3, considered the most relevant and in the database of EnANPAD, the most important event in the field of Administration, to investigate the concept and application of this term in national surveys. The development process for this study consisted of two stages: systematic and descriptive analysis. As a result, the research has shown the approach to the topic MO on national basis as a construct, as application and has shown existing limitations to memory settings. Studies on the subject are relatively recent in Brazil, which enables opportunities empirical or non-empirical and can contribute to the elaboration of constructs and applications nationwide.
\end{abstract}

Keywords: Organizational memory . Bibliometric technique . Knowledge management .

\section{REFERÊNCIAS}

ACKERMAN, M.S.: HALVERSON C. Organizational memory as objects, processes and trajectories: an examination of organizational memory in use. Computer Supported Cooperative Work, Kluwer Academic Publishers. Dordrecht, The Netherlands, v.13, n.2, p.155-189, 2004.

ARAÚJO, C. A. A. Bibliometria: evolução histórica e questões atuais. Em Questão, Revista da Faculdade de Biblioteconomia e Comunicação da UFRGS, Porto Alegre/RS, Brasil, v. 12, n. 1, p. 11-32, 2006.

ANDERSON, M.; SUN, P. What have scholars retrieved from Walsh an Undgson (1991)? A citation context study. Management Learning, SAGE, Thousand Oaks, USA, vol. 41, n. 2, p. 131-145, 2010. 
BORNEMANN, Manfred et al. An illustrated guide to knowledge management. Wissens management Forum, Graz, Áustria, 2003. Disponível em: <http://www.wm-forum.org>. Acesso em: 10 de out. de 2013.

COORDENAÇÃO DE APERFEIÇOAMENTO DE PESSOAL DE NÍVEL SUPERIOR. Qualis Pedriódicos. Brasil, 2012. Disponível em: <http://www.capes.gov.br/avaliacao/qualis>. Acesso em: 10 de out. de 2013.

CHANG, Jaegyong; CHOI, Byounggu; LEE, Heeseok. An organizational memory for facilitating knowledge: an application to e-business architecture. Expert Systems with Applications, Elsevier, USA, v.26, p.203-215, 2004.

DIENG-KUNTZ, Rose; MATTA, Nada (Eds.). Knowledge Management and Organizational Memories. New York: Springer Science, 2002. XXIV, 216 p. Disponível em: <http:// download.springer.com/static/pdf/729/bfm\%253A978-1-4615-0947-9\%252F1.pdf?au th66=1382009689_79d9ddb2d361e24627b2c4425cdb4e32\&ext=.pdf>. Acesso em:10 set. 2013.

DRUCKER, Peter F. Post-capitalist society. New York: Harper Collins, 1993.

GRANT, Robert M. Toward a knowledge-based theory of the firm. Strategic Management Journal, JSTOR,USA, (Winter Special Issue) v.17, pp.109-122, 1996.

JACKSON, Paul. Transactive directories of organizational memory: Towards a working data model. Information \& Management. Elsevier, USA, v.49, p.118-125, 2012.

KOGUT, Bruce; ZANDER, Udo. Knowledge of the firm, combinative capabilities, and the replication of technology. Organization Science. JSTOR, USA, v.3, n. 3, p.383-397, ago. 1992.

LOPÉZ, Suzana Pérez; PEÓN, José Manoel Montes; ORDÁS, Camilo José Vazquez. Organizational learning as a determining factor in business performance. The Learning Organization, Emerald, Bingley,UK, vol. 12, n. 3, p. 227-245, 2005.

NONAKA, Ikujiro; TAKEUCHI, Hirotaka. Criação de conhecimento na empresa. Rio de Janeiro: Campus, 1997.

NONAKA, Ikujiro; TOYAMA, Ryoco. A firm as a dialectical being: towards a dynamic theory of a firm. Industrial and Corporate Charge, ICC Association, Oxford, UK, v.11, p.995-1009, 2002. 
SANTOS, J. L. S.; MALDONADO, U. M.; DOS SANTOS, R. N. M. Mapeamento das publicações acadêmico-científicas sobre memória organizacional. In:ENCONTRO NACIONAL DA ASSOCIAÇÃO NACIONAL DE PÓS-GRADUAÇÃO E PESQUISA EM ADMINISTRAÇÃO - EnANPAD, 35. , 2011, Rio de Janeiro, Brasil Anais... Rio de Janeiro: ANPAD, 2011.

SANTOS, Raimundo N. M.; KOBASHI, Nair Y. Bibliometria, cientometria, infometria: conceitos e aplicações. Ciência da Informação, Brasília, Brasil, v. 2, n. 1, p. 155-172, 2009.

SASIETA, Héctor A. M.; BEPPLER, Fabiano D.; PACHECO, Roberto C. dos S. A Memória Organizacional no Contexto da Engenharia do Conhecimento. DataGramaZero - Revista de Informação, Brasil, v.12 n.3 ago, 2011. Disponível em: <http://www.dgz.org.br/ ago11/Art_06.htm> Acessado em 12 de out de 2013.

STEIL, Andrea V.; SANTOS, Jane L. Building conceptual relations between organizational learning, knowledge, and memory. International Journal of Business and Management Tomorrow. Toronto, Canada, v.2, n.2, Feb. 2012.

Stein, Eric W. Organizational memory: review of concepts and recommendations for management. International Journal of Information Management, Elservier, USA, v. 15, n.2, p. 17-32, 1995.

SUTTON, David; PATKAR, Vivek. CommonKADS analysis and description of a knowledge based system for the assessment of breast cancer. Expert Systems with Applications, Elsevier, USA, v. 36, n. 2, part 1, p. 2411-2423, mar. 2009.

TASCA, Jorge E. et al. An approach for selecting a theoretical framework for the evaluation of training programs. Journal of European Industrial Training, Emerald, Bingley, UK, v. 34, p. 631-655, 2010. Disponível em: <http://dx.doi. org/10.1108/03090591011070761>. Acesso em 10 de out. de 2013.

WALSH, J.; UNGSON, G. Organizational memory. Academy of management review, JSTOR,USA, v. 16, n. 1, p. 57-91, 1991.

WU, Liang-Chuan; ONG, Chorng-Shyong; HSU, Yao-Wen. Knowledge-based organization evaluation. Decision Support Systems, ACM Digital Library, Amsterdam, The Netherlands, v. 45, n. 3, p. 541-549, jun. 2008. 


\section{SOBRE OS AUTORES}
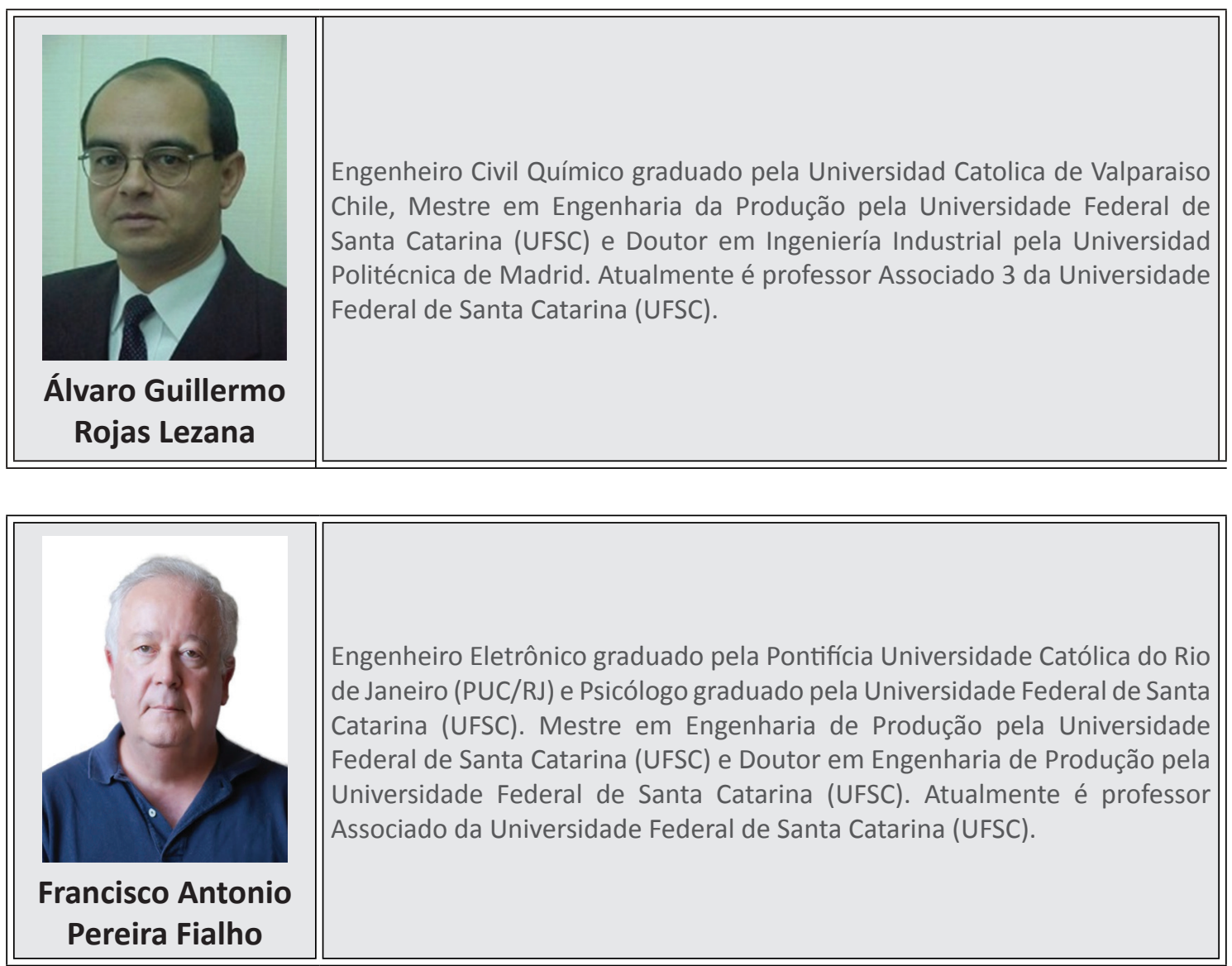

Administradora graduada pelo Centro Integrado de Palmas/PR. Mestre em
Engenharia e Gestão do Conhecimento pela Universidade Federal de Santa
Catarina (UFSC). Doutoranda em Engenharia e Gestão do Conhecimento
pela Universidade Federal de Santa Catarina (UFSC). Atualmente é designer
instrucional na Universidade Federal de Santa Catarina (UFSC).



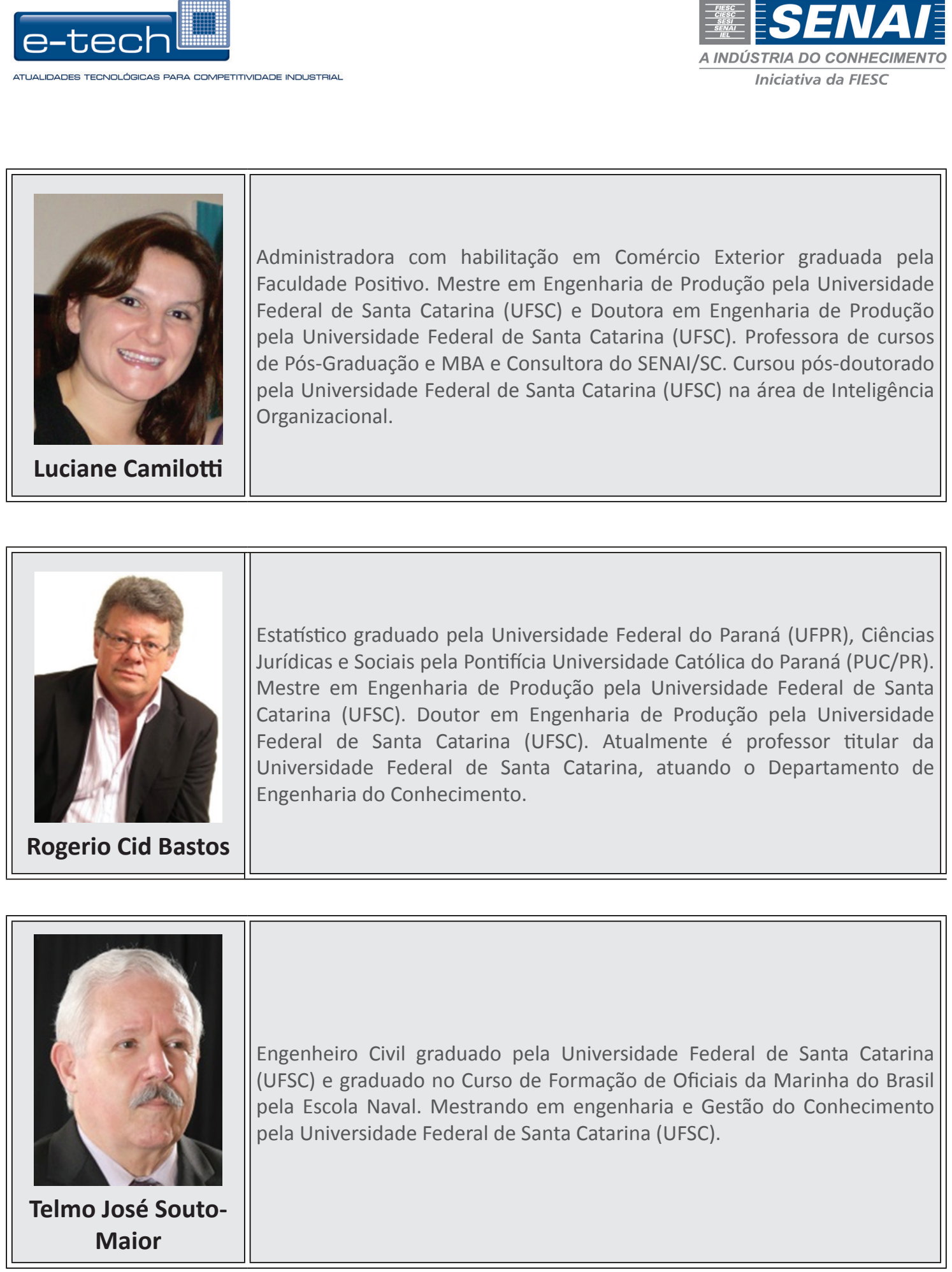

Originais recebidos em: 18/07/2013

Texto aprovado em: 15/10/2013 